\title{
Possibility of Observing Nonexponential Decays in Autoionizing States
}

\author{
Cleanthes A. Nicolaides and Donald R. Beck \\ Theoretical Chemistry Institute, National Hellenic Research Foundation (E.I.E.) Athens $501 / 1$, Greece
}

(Received 22 October 1976)

\begin{abstract}
A rigorous evaluation of the time evolution amplitude $G(t)$ for an isolated autoionizing state has yielded the result

$$
|G(t)|_{t \rightarrow \infty}^{2}=e^{-\Gamma t / \hbar}+\hbar^{2} / \pi^{2}\left(4 E^{2}+\Gamma^{2}\right) t^{2},
$$

where $E$ is the energy above the ionization threshold and $\Gamma$ the width. For states with lifetimes in the region $10^{-8}-10^{-11}$ sec near the ionization threshold, the nonexponential decay corrections are sufficiently large to suggest that perhaps it is possible to test the validity of the fundamental exponential "law" within the realm of atomic physics.
\end{abstract}

Resonance peaks observed in a variety of particle-atom or photon-atom scattering can be treated from a time-dependent point of view as being caused by nonstationary (autoionizing) states which decay into an electronic continuum. ${ }^{1-3}$ This approach assumes the creation of an initially completely localized state at $t=0$ (provided that $\Delta E$ $>\Gamma$, where $\Delta E$ is the width of the excitation wave packet and $\Gamma$ the decay width) which mathematically can be described by a square integrable $N$ electron wave function which is a solution of the Schrödinger equation in the continuum under imposed boundary conditions of square integrability. The important observed parameters then emerge naturally from the time evolution of this state.

The emphasis on the time dependence of these phenomena has led us to the following question: How do atomic systems evolve in time if they are assumed to be in a given nonstationary state at $t=0 ?$ As we will show below, it turns out that, for certain relativistically (mainly) autoionizing states, the corrections to the well-accepted exponential law which characterizes dissipative processes of isolated systems might be large enough to be observable.

Currently, there is considerable interest in measuring widths of autoionizing (Auger) states decaying via relativistic or Coulomb interactions. ${ }^{4}$ The experiments required to test the possible existence of the herein-derived nonexponential decays (NED) are of the intensity-vs-time measurement type.

We consider an isolated resonance. Our main physical arguments on the properties of autoionizing states, considered as decaying states, follow Refs. 1 and 2. A formal and general theory of decay processes has been given in the book of Goldberger and Watson. ${ }^{5}$ At $t=0$, the atom is assumed to be at a localized state $\left|\Psi_{0}\right\rangle$. Of course, the time $t=0$ cannot be defined exactly experimentally because of the finite time of excitation. However, this fact simplifies the final result of the decay analysis - at least in the ideal case. Because of the long-lived character of the excited states which might show NED and in accordance with the motion of decay, this Letter does not consider the effect of the preparation step on the time evolution of the autoionizig states, i.e., they are treated as though they forgot from whence they came.)

We are interested in the properties of the amplitude

$$
G(t)=\left\langle\Psi_{0}\left|e^{-(i / \hbar) H t}\right| \Psi_{0}\right\rangle,
$$

which is proportional to the number of atoms still in the original state after time $t$. As is well known, ${ }^{1,5} G(t)$ can be written as

$$
G(t)=\frac{1}{2 \pi i} \oint\left\langle\Psi_{0}|R(z)| \Psi_{0}\right\rangle e^{-(i / \hbar) z t} d z,
$$

where for $t>0$, the contour reduces to a line parallel to the real axis with $\operatorname{Im} z>0 . R(z)$ is the resolvent operator defined as

$$
R(z)=1 /(z-H) \text {. }
$$

Let

$$
R_{0}(z)=1 /\left(z-H_{0}\right),
$$

where

$$
H_{0} \Psi_{0} \equiv(H-V) \Psi_{0}=E_{0} \Psi_{0} .
$$

Then

$$
R(z)=R_{0}(z)+R_{0}(z) V R_{0}(z)+R_{0}(z) V R_{0}(z) V R(z) .
$$

From Eq. (6), it follows that

$$
\left\langle\Psi_{0}|R(z)| \Psi_{0}\right\rangle \equiv G^{d}(z)=\left[z-E_{0}-A(z)\right]^{-1},
$$


where

$$
A(z) \equiv\left\langle\Psi_{0}\left|V R_{0}(z) V\right| \Psi_{0}\right\rangle .
$$

$A(z)$ can be called the "self-energy" of the autoionizing state. Therefore, it is seen that the time evolution amplitude depends on (a) the properties of $G^{d}(z)$ and (b) the contour of integration imposed by the physics of autoionization.

We consider (a) first: The analytic properties of $G^{d}(z)$ on the various Riemann sheets depend on the solution of the transcendental equation

$$
z-E_{0}-A(z)=0 \text {. }
$$

Incidentally, if $\operatorname{Im} A(z)=0$ for some real $z$, either because of symmetry or accidentally, then a "bound state in the continuum" (BSC) appears since the resolvent has a pole on the real axis. (This is a case of a point spectrum superimposed on the continuous spectrum. A condition for this to occur is for the resolvent to be unbounded and defined on a set which is not dense in Hilbert space. ${ }^{3}$ )

Unfortunately, it is not easy to understand the analytic properties of $A(z)$ completely. However, in accordance with the physics of autoionization (e.g., for autoionizing states decaying relativistically, experimental evidence suggests that narrow widths are energy independent), we will assume that in the vicinity of $E_{0}, A(z)$ is analytic and essentially energy independent. Thus we take

$$
A(z) \approx A\left(E_{0}\right)=\Delta\left(E_{0}\right)-(i / 2) \Gamma\left(E_{0}\right) .
$$

Therefore, $G^{d}(z)$ has a pole at

$$
z_{0} \equiv E_{0}+A\left(E_{0}\right) \text {. }
$$

The negative imaginary part guarantees convergence of the integral of Eq. (2) in accordance with the notion of decay. The Green function $G^{d}(z)$ has a cut on the real axis above the ionization threshold but is analytic everywhere else on the physical sheet. Thus, the pole must be on the second Riemann sheet, just below the real axis, in order to have physical significance. In this work we have assumed that it is this pole that is responsible for the observable behavior of $G(t)$. Thus we take

$$
\left.\left\langle\Psi_{0}|R(E+i 0)| \Psi_{0}\right\rangle \underset{\text { continuation }}{\text { analytic }} \frac{1}{z-z_{0}}\right|_{\operatorname{Im} z<0} .
$$

Now we consider (b), the path of integration. Using the complete states of $H, \mathrm{Eq}$. (1) can be written as

$$
\begin{aligned}
G(t)= & \sum_{n} \exp \left[(-i / \hbar) E_{n} t\right]\left|\left\langle\Psi_{0} \mid \Psi_{n}\right\rangle\right|^{2} \\
& +\int_{I_{1}}^{\infty} d E \exp [-(i / \hbar) E t]\left|\left\langle\Psi_{0} \mid \Psi(E)\right\rangle\right|^{2},
\end{aligned}
$$

where, in general, $\left\langle\Psi_{0} \mid \Psi_{n}\right\rangle \neq\left\langle\Psi_{0} \mid \Psi(E)\right\rangle \neq 0,\langle\Psi(E)|$ $\times\left|\Psi\left(E^{\prime}\right)\right\rangle=\delta\left(E-E^{\prime}\right)$. $I_{1}$ is the first ionization threshold and $\Psi_{0}$ is embedded in the corresponding continuum. Expression (13) includes adjoint (time-reversed) states.

The asymmetry of the integral in (13) and the reality of the function $g(E) \equiv\left|\left\langle\Psi_{0} \mid \Psi(E)\right\rangle\right|^{2}$ for $E \geqslant I_{1}$ are sufficient to prove that $G(t)$ cannot decay exponentially at all times..$^{3,6}$ It has also guided us in the choice of the contour since energy restrictions do not allow decay in the region $\operatorname{Re} z<I_{1}$. Thus, the contour of integration is chosen as shown in Fig. 1, where the pole is depicted on the second Riemann sheet.

Using Eqs. (2) and (12) we consider the line integral on the real axis:

$$
\begin{aligned}
G(t) & =\frac{1}{2 \pi i} \int_{C_{1}} \frac{\exp [(-i / \hbar) z t]}{z-z_{0}} d z \\
& =\exp \left[(-i / \hbar) z_{0} t\right]-\frac{1}{2 \pi i}\left[\int_{C_{2}}+\int_{C_{3}}\right]
\end{aligned}
$$

As $z \rightarrow \infty, \int_{C_{2}}$ vanishes and the remaining righthand side integral can be identified with the integral 5.1.28 in the National Bureau of Standards tables of mathematical functions. ${ }^{7}$ Thus,

$$
G(t)=\exp \left[(-i / \hbar) z_{0} t\right]\left[1-\frac{1}{2 \pi i} \operatorname{Ei}\left((-i / \hbar) z_{0} t\right)\right]
$$

where $\operatorname{Ei}(u)$ is the exponential integral.

Equation (15) is valid for all $t$. For $t \rightarrow \infty$ we use the lowest-order asymptotic expansion for $\operatorname{Ei}(u)$

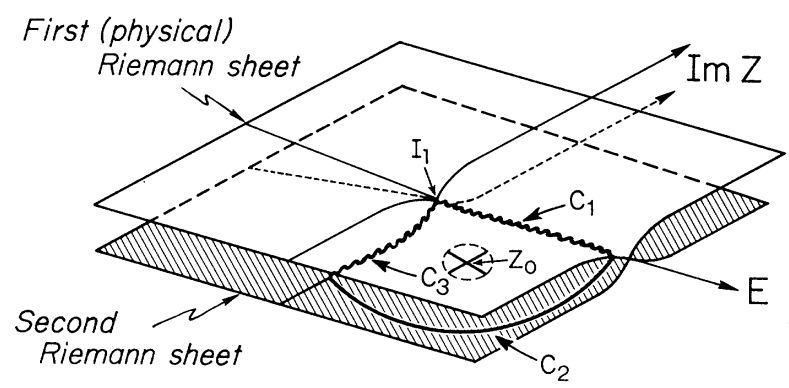

FIG. 1. Integration path for evaluating the amplitude $G(t)$ [Eq. (14)]. The pole $z_{0}$ is put on the second Riemann sheet. 
and obtain for the decay rate $|G(t)|^{2}$

$$
|G(t)|^{2}=e^{-(\Gamma / \hbar) t}+\frac{\hbar^{2}}{\pi^{2}\left(4 E^{2}+\Gamma^{2}\right) t^{2}} .
$$

In deriving Eq. (16), the oscillatory terms have been dropped because at the observation point an averaging must take place since, because of the finite time of excitation (which, however, is still smaller than $\hbar / \Gamma)$, the instant $t=0$ is not well defined.

For an isolated resonance, Eq. (16) represents a rigorous replacement of the exponential "law" for large $t$. The physical approximation employed was Eq. (12) and the physical origin of the deviation is the existence of the lower bound on the energy spectrum.

The analytic expression (16) allows one to evaluate trivially the magnitude of NED given a pair $E$ and $\Gamma$. In order to calculate $|G(t)|^{2}$ for all $t$ and a large number of values of $E$ and $\Gamma$, we have derived an alternative expression by breaking the line integral $\int_{C_{3}}$ into its real and imaginary parts and integrating each piece numerically. The result is

$$
|G(t)|^{2}=e^{-(\Gamma / \hbar) t}\left[1+\frac{I_{a}^{2}+I_{b}^{2}}{4 \pi^{2}}\right]
$$

where

$$
\begin{aligned}
& I_{a} \equiv \int_{-1 / 2}^{\infty} \frac{\omega d \omega e^{-\omega x}}{\omega^{2}+R^{2}} \\
& I_{b} \equiv R \int_{-1 / 2}^{\infty} \frac{d \omega e^{-\omega x}}{\omega^{2}+R^{2}}
\end{aligned}
$$

$R=E / \Gamma$ and $X=\Gamma t / \hbar$, the number of lifetimes

The NED corrections represent a slowing down of the rate of outgoing electrons as can be seen from Eq. (16) and Fig. 2, where a range of $R$ values has been selected to represent nonrelativistically allowed and forbidden decays. Obviously $R$ must remain large enough so that the concept of an initially localized and isolated autoionizing state remains valid. It should be noted that in the limit $t \rightarrow \infty$ expression (17) also reduces to Eq. (16) (the proof is given in Ref. 3).

Although only a few atomic spectra are known accurately above the ionization threshold, there is sufficient evidence for metastable states which can decay via spin-orbit or spin-spin interactions $\left(\Gamma \sim 10^{-7}-10^{-11} \mathrm{eV}\right)$ and are very close $\left(E \sim 10^{-1}-\right.$ $10^{-2} \mathrm{eV}$ ) to the ionization threshold (e.g., in O I, $\mathrm{Cu}$ I, Mo I, Sn I, SbI). In general, such situations will arise when a Rydberg series crosses the threshold of a different (nonrelativistic) symme-
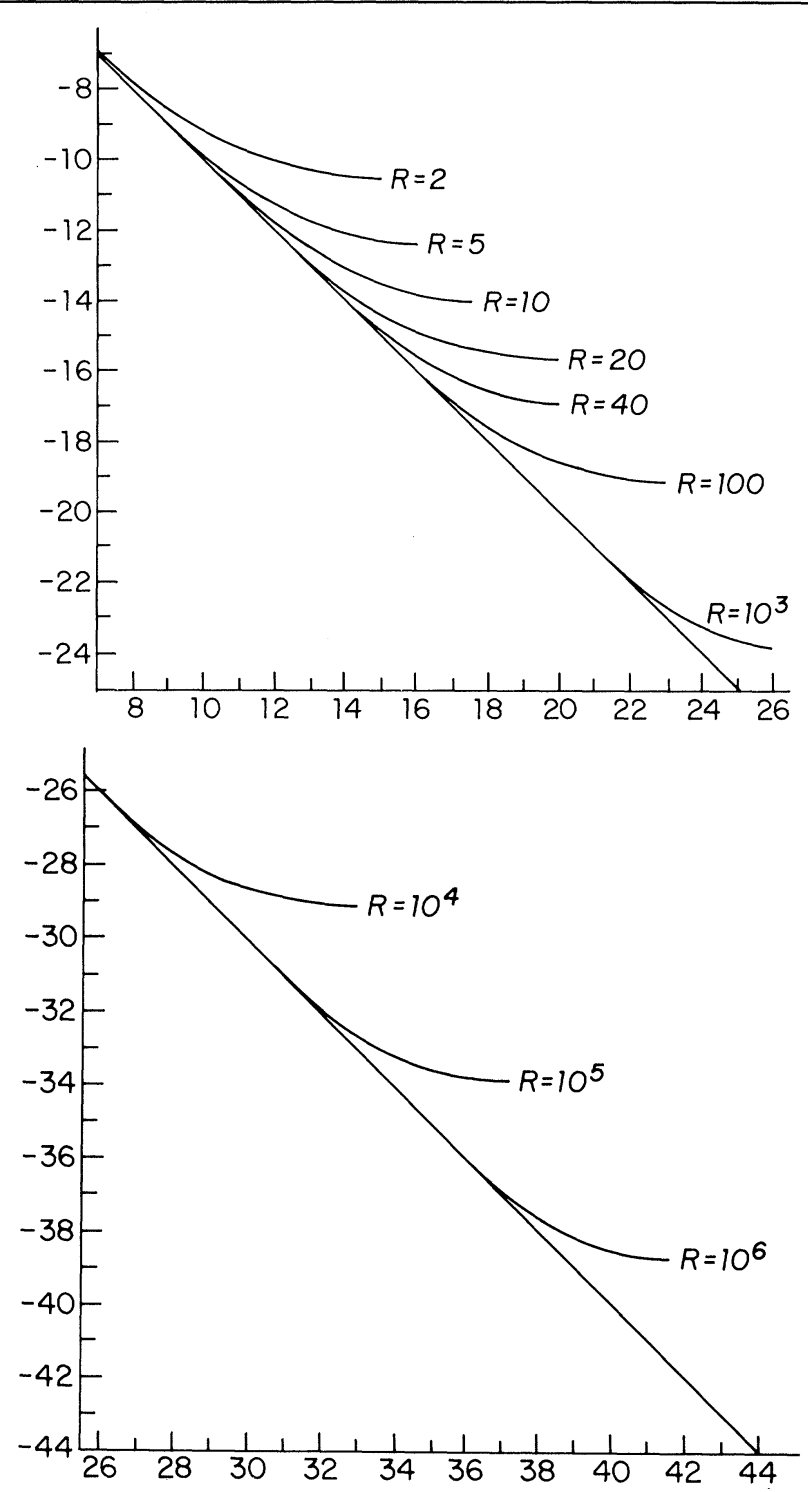

FIG. 2. Plot of

$$
\ln |G(t)|^{2}=-x+\ln \left[1+\frac{I_{a}^{2}(x, R)+I_{b}^{2}(x, R)}{4 \pi^{2}}\right]
$$

[see Eq. (17)]. $x=\Gamma t / \hbar=$ number of lifetimes. $R=E / \Gamma$. The straight line is the exponential law. NED is larger for small $R$.

try. The choice, of course, of relativistically decaying states facilitates a measurement of intensity as a function of time with existing devices because of their long lifetimes.

Orders of magnitude of NED can easily be read off Fig. 2. As an example, we use the following extreme case: For $E=10^{-3} \mathrm{eV}$ and $\Gamma=10^{-5} \mathrm{eV}$, NED is $20 \%$ of the magnitude of the exponential term after about 18 lifetimes. If $10^{15}$ atoms can 
be excited to that state, then after 18 to 28 lifetimes the number of excited atoms is between $10^{7}$ and $10^{3}$, a number which in principle can produce a detectable signal. (We point out, however, that detection of emitted electrons of very low energies is by no means a trivial experimental procedure.)

We close by bringing to attention the fact that our NED formula is different from the ones found in the work of Goldberger and Watson. ${ }^{5,8}$ Our approach consists of taking $G^{d}(z)=1 /\left(z-z_{0}\right)$ with 0 $\leqslant \operatorname{Re} z<\infty$ and $\operatorname{Im} z<0$, the emphasis essentially being on the fact that the integration over the energy axis must start from $I_{1}$ (and not from $-\infty$ ) and on the expectation that for autoionizing states and especially those decaying via relativistic interactions, the observable time evolution is due to the behavior of $G^{d}(z)$ around $E_{0}$ only.

In conclusion, the theoretical predictions derived above suggest that there is a class of autoionizing states for which NED effects might not be prohibitively small for observation. Thus, they provide a unique opportunity to test in atomic physics the validity of a fundamental "law" of nature. A theory and application of the physical and mathematical properties of autoionizing states and their electronic structure is presented in a longer paper. ${ }^{3}$

${ }^{1}$ C. A. Nicolaides, Phys. Rev. A 6, 2078 (1972).

${ }^{2}$ C. A. Nicolaides, Nucl. Inst. Methods 110, 231 (1973).

${ }^{3}$ C. A. Nicolaides and D. R. Beck, Phys. Rev. A (to be published).

${ }^{4}$ E.q.. P. Feldman and R. Novick, Phys. Rev. 160 , 143 (1967); H. G. Berry, J. Desesquelles, and M. Dufay, Phys. Lett. 36A, 237 (1971); I. A. Sellin, D. J. Pegg, P. M. Griffin, and W. W. Smith, Phys. Rev. Lett. 28, 1229 (1972); K. Siegbahn et al., ESCA Applied to Free Molecules (North-Holland, Amsterdam, 1969); W. Mehlorn, Phys. Fenn. $\underline{9}, 223$ (1974).

${ }^{5}$ M. L. Goldberger and K. M. Watson, Collision Theory (Wiley, New York, 1964).

${ }^{6}$ C. A. Nicolaides and D. R. Beck, in Beam-Foil Spectroscopy, edited by I. Sellin and D. Pegg (Plenum, New York, 1976), Vol. 1, p. 77.

${ }^{7}$ Handbook of Mathematical Functions, edited by M. A. Abramowitz and I. Stegun, National Bureau of Standards, Applied Mathematics Series No. 55 (U. S. Government Printing Office, Washington, D. C., 1964).

${ }^{8}$ M. L. Goldberger and K. M. Watson, Phys. Rev. 136, B1472 (1964).

\title{
High-Resolution Atomic Beam Study of the Helium Excimer Potentials $\operatorname{He}_{2}\left(A, C^{1} \Sigma_{\mathrm{u}, \mathrm{g}}{ }^{+}\right)$
}

\author{
Bernhard Brutschy and Hellmut Haberland \\ Fakultät für Physik der Universität Freiburg, Freiburg, Germany \\ (Received 14 January 1977)
}

\begin{abstract}
The long-range parts of the $A, C{ }^{1} \Sigma_{\mathrm{u}_{\mathrm{g}}}{ }^{+}$excimer potentials of the $\mathrm{He}_{2}$ molecule have been accurately dtermined from high-resolution differential-cross-section measurements at the relative kinetic energy range from 18 to $140 \mathrm{meV}$. The barrier height of the intermediate maximum of the $A^{1} \Sigma_{\mathrm{u}}{ }^{+}$potentials is $47_{-1}^{+2} \mathrm{meV}$ at an internuclear distance of $3.14 \pm 0.05 \AA$. The long-range parts of the potential from the recent ab initio calculation by Guberman and Goddard is always 5 to $20 \mathrm{meV}$ higher than the potential determined from our experiments.
\end{abstract}

The lowest electronically excited or excimer states of the rare-gas diatomic molecules have recently been studied very intensively because of the possibility of building tunable uv lasers. The simplest case is the excimer states of the $\mathrm{He}_{2}$ molecule, from which lasing has not been observed so far, but which are of considerable theoretical and practical interest. ${ }^{1,2}$

We have studied the long-range parts of the $A^{1} \Sigma_{\mathrm{u}}{ }^{+}$and $C^{1} \Sigma_{\mathrm{g}}{ }^{+}$helium excimer potentials in a crossed-atomic-beam experiment. The experimental details have been described earlier..$^{3-6}$ Briefly, helium atoms of a supersonic beam of variable kinetic energy (16-250 meV) and very good velocity resolution ( 1 to $8 \%$ ) is excited by coaxial electron impact to the two metastable states $\left(1 s 2 s, 2^{1} S\right.$ and $\left.2^{3} S\right)$. The singlet state can be quenched optically. This beam is crossed at a right angle with a beam of ground-state $\mathrm{He}$ atoms, and the angular distribution of the excited helium $\left(\mathrm{He}^{*}\right)$ atoms is measured.

Figure 1 shows the experimental results for six different kinetic energies. The scattered flux of $\mathrm{He}\left(2^{1} S\right)$ atoms is plotted against the lab scattering angle. The center-of-mass (c.m.) scattering angle is obtained by multiplying the lab angle by a factor of 2. All data points are normalized with the reading of a stationary monitor detector, 